which appear eosinophilic if the cytoplasm is plentiful and rich in mitochondria, or basophilic if more scanty with fewer mitochondria. Clear cell and chromophilic cell types of renal cell carcinomas co-express cytokeratin and vimentin and are thought to be derived from the proximal convoluted tubule. ${ }^{23}$ These cell types are commonly seen together in any one tumour. De-differentiated 'sarcomatoid' spindle cell forms of renal cell carcinomas are also seen. Some tumours are composed of pale chromophilic cells containing microvesicles but little glycogen or lipid, which express cytokeratin but not vimentin and are thought to be derived from the intercalated cells of the collecting duct.

Solid, acinar, trabecular, or tubulo-papillary architectural patterns may also be seen, frequently in combination, in any one tumour. ${ }^{1}$ Secondary changes such as cystic degeneration, haemorrhage and necrosis are also common in renal cell carcinomas.

There is some association between cell type and architectural pattern with the clear cell and chromophobic tumours producing a more solid compact pattern, whereas the chromophilic cell types usually produce a tubulopapillary pattern, with papillae containing fibrovascular cores. ${ }^{1}$ The pure papillary variant contains prominent numbers of macrophages with foamy cytoplasm in the papillary cores.

In the present case the tumour described is a chromophilic renal cell carcinoma (Fuhrman grade $2, \mathrm{~T} 2 \mathrm{~N} 0 \mathrm{Mx}$, stage 2$)^{4}$ which is poor in mitochondria for the degree of eosinophilia present and has an unusual microtubular growth pattern and myxoid stroma. The tumour was monomorphic throughout. The absence of other degenerative changes suggests that the myxoid stroma is a primary component of the tumour.

The prognosis of renal cell carcinomas depends on the nuclear grade and the stage, and is independent of the tumour cell type and architecture, except for the sarcomatoid and papillary tumours which fare worse. ${ }^{5}$ The clinical significance of the unusual tumour pattern described here is doubtful, but it has not been reported previously. The unusual appearance is a potential source of diagnostic errorother myxoid tumours, such as myxoid chondrosarcoma or leiomyosarcoma, may appear similar on initial microscopic inspection-but immunohistochemistry will provide the diagnosis.

1 Thoenes W, Storkel S, Rumpelt HJ. Histopathology and classification of renal cell tumors (adenomas, oncocytomas, and carcinomas). The basic cytological and histopathological es Pract cal elements and their

2 Pitz S, Moll R, Storkel S, Thoenes W. Expression of intermediate filament proteins in subtypes of renal cell carcinomas and in renal oncocytomas. Lab Invest 1987;56:642-53.

3 Gerharz C-D, Moll R, Storkel S, Ramp U, Thoenes W, Gabbert HE. Ultrastructural appearances and cytoskeletal architecture of the clear, chromophilic and chromophobe types of human renal cell carcinoma in vitro. Am $¥$ Pathol types of human renal cell carcinoma in vitro. Am f Pathol $\mathbb{Q}$

4 Furhman SA, Lasky LC, Limas C. Pathological significance of morphological parameters in renal cell carcinoma. $A m \mathcal{F}$ Surg Pathol 1982;6:655-63.

5 Nasady T, Bane BL, Silva FG. Chapter 41. Adult renal diseases. Sternberg SS, Antonioli DA, Carter D, Mills SE, Oberman HA, eds. Diagnostic surgical pathology. 2nd edn. New York: Raven Press, 1994:1645-739.

\title{
Helicobacter pylori antibody titres in serum, plasma and successively thawed specimens: implications for epidemiological and clinical studies
}

Department of Public

Health and

Community Medicine,

Ballarat Base Hospital,

University of

Melbourne

D C Pearce

H G Peach

Epidemiology and Biostatistics Unit S J Farish

Correspondence to: Ms D C Pearce, Department of Public Health and Community Medicine, Ballarat Base Hospital, PO Box 577, Ballarat VIC 3353 Australia.

Accepted for publication 4 September 1996

\author{
D C Pearce, H G Peach, S J Farish
}

\begin{abstract}
Agreement between Helicobacter pylori IgG antibodies measured using the Pyloriset EIA-G kit in serum, plasma and successively thawed specimens was studied and the implications for epidemiological and clinical studies assessed. Plasma titres may differ from serum titres by $-6 \%$ to $+8 \%$ and therefore may be substituted for serum. The change in titre around the cut off value was -0.31 (se $=5.7, p=0.96$ ) per thaw. The estimated maximum drop after three thawings, 34.5 , would result in only a small decrease in sensitivity (1.3\%).
\end{abstract}

For qualitative epidemiological studies, $\stackrel{\mathscr{\infty}}{+}$ this additional misclassification rate is 0 relatively small. However, positive titres did reduce over successive thawings, with

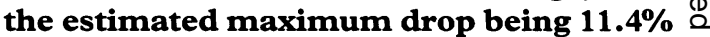
per thaw. Therefore, thawing does need to be considered as a contributing factor 8 when interpreting titre drops in eradication trials. Baseline and follow up speci- 흘 mens from clinical studies should be $\supsetneq$ thawed once only and tested concurrently. (F Clin Pathol 1996;49:1017-1019)

Keywords: Helicobacter pylori, epidemiology, serology. 
The ability to measure antibodies directed against Helicobacter pylori in serum on large numbers of subjects relatively inexpensively has made epidemiological studies of $H$ pylori infection possible. The manufacturer of the enzyme immunoassay kit used to measure $H$ pylori IgG antibody titres in this study recommends that when evaluating the efficacy of eradication treatment, the pretreatment and follow up specimens be tested concurrently to improve accuracy. This has important implications for epidemiological as well as clinical studies of $H$ pylori infection. Specimens taken in different places (as in ecological and prevalence studies) or on several occasions (as in prospective studies or follow up of treatment trials) would need to be frozen and transported to, and/or stored in, a central laboratory until they can be thawed and tested together. This should be possible with careful planning. However, researchers often find it convenient to thaw and test specimens which have been stored during epidemiological studies of diseases other than $H$ pylori infection, particularly major prospective studies. Such specimens may have been thawed and refrozen on several occasions prior to testing for $H$ pylori antibodies, which is something that the manufacturer's instructions recommend avoiding.

Although numerous studies report having tested serum specimens taken in different places or on several occasions, none seem to have assessed the effect of freezing and thawing the specimens on $H$ pylori antibody titres.

Serum samples have often been used in enzyme immunoassays. However, it may be convenient to test plasma samples for antibodies directed against $H$ pylori in parallel with other biochemical tests. Here, we report on the implications of testing plasma as an alternative to serum and of using successively thawed and refrozen serum specimens for the measurement of $H$ pylori antibody titres during epidemiological and clinical studies.

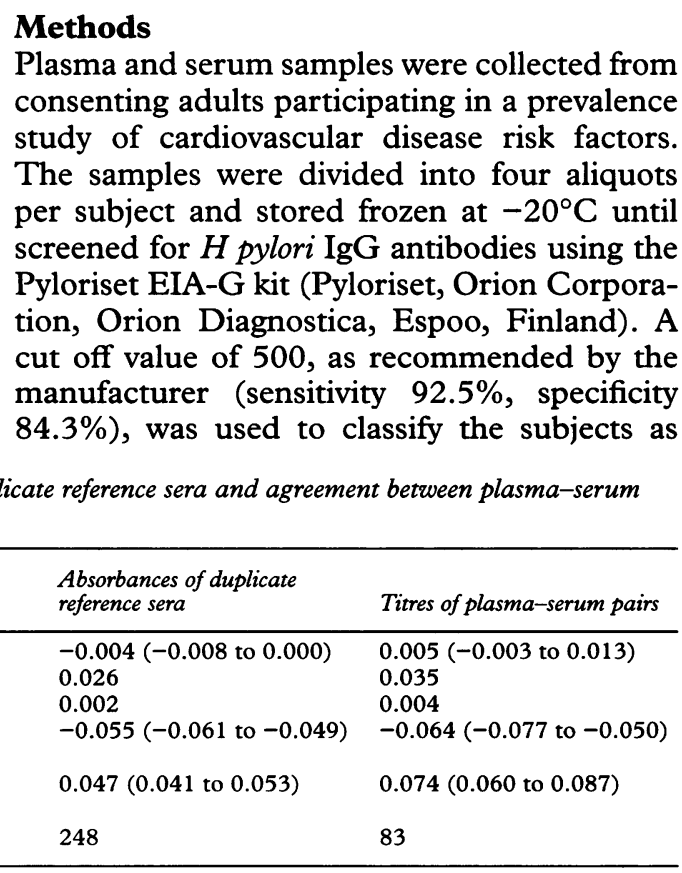

positive or negative. ${ }^{1}$ Subsequently, paired plasma and serum samples from a random 38 $H$ pylori positive and $45 \mathrm{H}$ pylori negative subjects were tested concurrently.

Aliquots of serum from a random six of the $H$ pylori positive and six of the $H$ pylori negative subjects were systematically thawed and refrozen up to three times giving up to four separate samples. Sets of thawed aliquots were tested concurrently for antibodies directed against $H$ pylori.

Titres of the plasma-serum pairs were determined from a standard curve, obtained by plotting mean absorbances of duplicate reference sera of specified antibody titre on semi-logarithmic graph paper. The repeatability of the absorbances of the duplicate reference sera and the agreement between titres in paired plasma and serum samples were assessed by the methods of Bland and Altman. ${ }^{2}$

Changes in titre value as a result of multiple thawings were assessed using a generalised linear models procedure to perform a repeated measures analysis with the number of thawings as a linear covariate. This enabled statistically significant changes in the titre to be detected. The model used was adjusted with a parameter for each individual subject.

\section{Results}

The differences in absorbance between duplicate reference sera and in titre between plasma-serum pairs were proportional to the means. Therefore, log-transformed data were used in assessing repeatability of duplicate reference sera and agreement between plasmaserum pairs.

The mean differences between the logtransformed absorbances of the duplicate reference sera and between the titres of plasma-serum pairs were negligible, as shown in table 1. The lower and upper limits of this agreement were calculated from the mean differences $\pm 1.96 \times$ SD of the differences and were narrow (table 1). The precision of these estimated mean differences and limits of agreement was determined from the $95 \%$ confidence intervals (CI). These intervals are narrow, suggesting the agreements both between the absorbances of the duplicate sera and between titres obtained from the plasmaserum pairs are acceptable.

When the limits of agreement were related to the original data by taking the antilogs, we estimated that about $95 \%$ of the duplicate reference sera absorbances will differ by less than 5\%. For the plasma-serum pairs, the titre for plasma samples may differ by $6 \%$ below or $8 \%$ above that obtained for serum samples. These differences are not considered to be clinically or epidemiologically significant. Hence, plasma may be substituted for serum when testing $H$ pylori antibodies using this EIA-G kit during epidemiological or clinical research.

For epidemiological studies, we were interested in the effect of multiple thawings on serum titres around the cut off value of the assay which might lead to an increase in the proportion of false negative results. Therefore, 
we excluded two subjects with high titres, 1900 and 3600 , well above the cut off value of 500 in the repeated measures analysis of this effect. The average change in titre (using untransformed data) detected among the remaining subjects was -0.31 per thaw (se $=5.7$, $p=0.96$ ). The maximum probable change per thaw was estimated (from the $95 \% \mathrm{CI}$ ) at -11.5 . Hence, after three thawings it would be possible for the titre to drop by as much as 34.5. This would cause the sensitivity of the test to decrease only slightly (estimated reduction $1.3 \%$ based on the receiver operator characteristic (ROC) curve produced by the manufacturer). This small decrease from $92.5 \%$ to $91.2 \%$ in the proportion of $H$ pylori positive subjects detected by the assay is not of any epidemiological significance.

For eradication trials we were interested in the effect of thawings on serum titres among all subjects who were $H$ pylori positive and who would normally be eligible for treatment. For these subjects the mean titre was 1245 and the average drop in titre detected by repeated measures analysis was -75.8 per thaw (se = $33.7 \mathrm{p}=0.04)$. The maximum probable drop per thaw was $11.4 \%$, which may be of clinical significance.

\section{Discussion}

Our results suggest that either plasma or serum, and specimens that have been stored frozen and subsequently thawed on several occasions, can be used for epidemiological research where a small additional misclassifica- tion rate can be tolerated. Specimens which have thawed accidentally during storage or transportation and been refrozen may also be usefully tested for $H$ pylori status.

Among all $H$ pylori positive subjects, the change in titre per thaw was statistically significant and the estimated drop could be as extreme as $11.4 \%$ per thaw. This needs to be taken into account when using a drop in titre as an indicator of eradication of $H$ pylori infection during follow up of treatment. Successful eradication of $H$ pylori infection is usually associated with a drop in titre of at least $60 \%$ of the pretreatment value, which generally occurs within 12 months. ${ }^{3}$ It is possible that multiple thawing of specimens could make a significant contribution to such a drop in titre. Thawing baseline and follow up specimens once only and testing all specimens concurrently would be prudent.

We thank Mr C Pearce and staff of the Department of Microbiology, Dorevitch Pathology, Ballarat Base Hospital, for thei technical assistance and use of facilities.

This study was funded by the Shepherd Foundation.

The Department of Public Health and Community Medicine is a member of the Victorian Public Health Consortium.

1 Granberg C, Mansikka A, Lehtonen O-P, Kujari $H$ Gronfors R, Nurmi $\mathrm{H}$, et al. Diagnosis of Helicobacter pylori infection by using Pyloriset EIA-G and EIA-A for detection of serum immunoglobulin $\mathrm{G}$ (IgG) and IgA antibodies. F Clin Microbiol 1993;31:1450-3.

2 Bland JM, Altman DG. Statistical methods for assessing agreement between two methods of clinical measurement Lancet 1986;i:307-10.

3 Kosunen TU, Seppälä K, Sarna S, Sipponen P. Diagnostic value of decreasing IgG, IgA, and IgM antibody titres afte eradication of H. pylori. Lancet 1992;339:893-5.

\title{
The order of draw of blood specimens into additive containing tubes does not affect potassium and calcium measurements
}

\author{
Department of \\ Medicine and \\ Therapeutics, \\ Western Infirmary, \\ Glasgow G11 6NT \\ A Majid \\ D C Heaney \\ N Padmanabhan

\section{Department of} \\ Biochemistry \\ R Spooner \\ Correspondence to: \\ Dr A Majid, \\ Department of Neurology, \\ Washington University \\ School of Medicine, \\ Campus Box 8111 , \\ 660 S Eucid \\ MO 63110-1093 \\ USA.
}

Accepted for publication 4 September 1996

\author{
A Majid, D C Heaney, N Padmanabhan, R Spooner
}

\begin{abstract}
The effect of order of draw when taking blood into tubes containing additive was investigated in 47 medical inpatients; 12 of these patients acted as a control group. The samples were analysed in the order in which they were withdrawn. The results of potassium and calcium concentrations did not differ significantly between groups. Manufacturers recommend a specific order of draw when taking blood using vacuum based blood collection systems, which are routinely used in many hospitals. The results of this study, how-
\end{abstract}

ever, show that order of draw has no effect on calcium or potassium concentrations. (F Clin Pathol 1996;49:1019-1020)

Keywords: order of draw, venepuncture, Vacutainer system, potassium concentration, calcium concentration.

The importance of correct collection and handling of blood specimens has been highlighted before. ${ }^{12}$ Calam and Cooper have suggested that the order of draw of blood specimens into tubes containing additive may affect measured serum potassium and calcium concentrations. ${ }^{3}$ In their small $(n=5)$ report they suggest that if 